\title{
Modeling and Simulation for High Energy Sub-Nuclear Interactions Using Evolutionary Computation Technique
}

\author{
Mahmoud Y. El-Bakry1', El-Sayed A. El-Dahshan'2,3, Amr Radi3 ${ }^{3,4}$, Mohamed Tantawy1, \\ Moaaz A. Moussa ${ }^{1,5}$ \\ ${ }^{1}$ Department of Physics, Faculty of Sciences, Ain Shams University, Cairo, Egypt \\ ${ }^{2}$ Egyptian E-Learning University, Giza, Egypt \\ ${ }^{3}$ Department of Physics, Faculty of Education, Ain Shams University, Cairo, Egypt \\ ${ }^{4}$ The British University in Egypt (BUE), Cairo, Egypt \\ ${ }^{5}$ Buraydah Colleges, East Qassim University, Buraydah, KSA \\ Email: moaaz2030@yahoo.com
}

Received 19 November 2015; accepted 10 January 2016; published 13 January 2016

Copyright (C) 2016 by authors and Scientific Research Publishing Inc.

This work is licensed under the Creative Commons Attribution International License (CC BY).

http://creativecommons.org/licenses/by/4.0/

c) (i) Open Access

\section{Abstract}

High energy sub-nuclear interactions are a good tool to dive deeply in the core of the particles to recognize their structures and the forces governed. The current article focuses on using one of the evolutionary computation techniques, the so-called genetic programming (GP), to model the hadron nucleus (h-A) interactions through discovering functions. In this article, GP is used to simulate the rapidity distribution $\left(\frac{1}{N} \frac{\mathrm{d} N}{\mathrm{~d} Y}\right)$ of total charged, positive and negative pions for $\mathbf{p}^{-}$-Ar and p--Xe interactions at $200 \mathrm{GeV} / \mathrm{c}$ and charged particles for p-pb collision at $5.02 \mathrm{TeV}$. We have done so many runs to select the best runs of the GP program and finally obtained the rapidity distribution $\left(\frac{1}{N} \frac{\mathrm{d} N}{\mathrm{~d} Y}\right)$ as a function of the lab momentum $\left(P_{L a b}\right)$, mass number $(\mathrm{A})$ and the number of particles per unit solid angle (Y). In all cases studied, we compared our seven discovered functions produced by GP technique with the corresponding experimental data and the excellent matching was so clear.

\section{Keywords}

Modeling, Simulation, Evolutionary Computation, Genetic Programming, Hadron-Nucleus Interaction, Rapidity Distribution 


\section{Introduction}

Evolutionary computation refers to a class of algorithms that utilize simulated evolution to some degree as a means to solve a variety of problems, from numerical optimization to symbolic logic. By simulated evolution, we mean that the algorithms have the ability to evolve a population of potential solutions such that weaker solutions are removed and replaced with incrementally stronger (better) solutions. In other words, the algorithms follow the principle of natural selection. Each of the algorithms has some amount of biological plausibility, and is based on evolution or the simulation of natural systems [1]-[8].

In 1990s, John Koza [9] [10] introduced the subfield called Genetic Programming. This is considered a subfield because it fundamentally relies on the core genetic algorithm created by Holland [11], and differs in the underlying representation of the solutions to be evolved. Instead of using bit-strings (as with genetic algorithms) or real-values (as is the case for evolutionary programming [1]-[7] or evolutionary strategies [1]-[7], genetic programming relies on S-expressions (program trees) as the encoding scheme.

Hadron-nucleus (h-A) interactions have been considered as a corner stone in high energy physics because of its theoretical and practical interesting features and also it is an intermediate state between hadron-hadron (h-h) and nucleus-nucleus (N-N) interactions. So, there are a lot of models that concern the study of the hadron structure [12]-[16] and the interactions between hadrons and nuclei such as the three-fireball model [17], quark models [18]-[20], fragmentation model [21]-[23] and many more.

In our previous works [24]-[27], our group studied the applications of artificial intelligence and the evolutionary computation techniques such as neural network, adaptive fuzzy inference system, genetic programming, genetic algorithm, hybrid technique model and many others to solve many complex (nonlinear) problems in high energy physics and showed best fitting with the corresponding experimental data in comparison with the conventional techniques.

The study of hadron-nucleus interaction at high and ultrahigh energy has been a subject of great interest to high energy physicist because the nuclei provide a number of unique physics opportunities which are not available in elementary particle collisions [28].

In this article, Genetic programming (GP) model has been used to discover a function that computes the rapidity distribution of created (total charged, positive and negative) pions for $\mathrm{p}^{-}$-Ar and $\mathrm{p}^{-}$-Xe collisions at 200 GeV/c [29]-[31] and charged particles for p-pb collision at 5.02 TeV [32]. The seven discovered functions produced by GP model show an excellent matching when they have been compared to the corresponding experimental data [29]-[32]. This article is organized as follows; Section 2 gives the definition and the outlines to the basics of the GP technique. Section 3 reviews the implementation of GP. Finally, the results and conclusions are provided in Sections 4 and 5 respectively.

\section{GP Outlines}

GP is defined as the biologically-inspired evolution of computer programs that solve a predefined task. For this reason, GP is nothing more than a genetic algorithm applied to the problem program evolution. Early GP systems utilized LISP (the original functional programming language, 1958) S-expressions (as shown in Figure 1), but more recently, linear GP systems have been used to evolve instruction sequences to solve user-defined programming tasks [2].

Since genetic programming manipulates programs by applying genetic operators (reproduction, crossover and mutation), a programming language should permit a computer program to be manipulated as data and the newly created data to be executed as a program. For these reasons, LISP was chosen as the main language for GP.

Evolving complete programs with GP is computationally very expensive, and the results have been limited, but GP does have a place in the evolution of program fragments. For example, the evolution of individual functions that have very specific inputs and outputs and whose behavior can be easily defined for fitness evaluation by GP. To evolve a function, the desired output must be easily measurable in order to understand the fitness landscape of the function in order to incrementally evolve it [1]-[7].

Consider the example shown in Figure 1. The population consists of two members, A and B. Using the crossover operator, a portion of $\mathrm{A}$ is grafted onto B; resulting in a new expression C. GP also utilizes the mutation operator as a way of extending the population to the search space. 


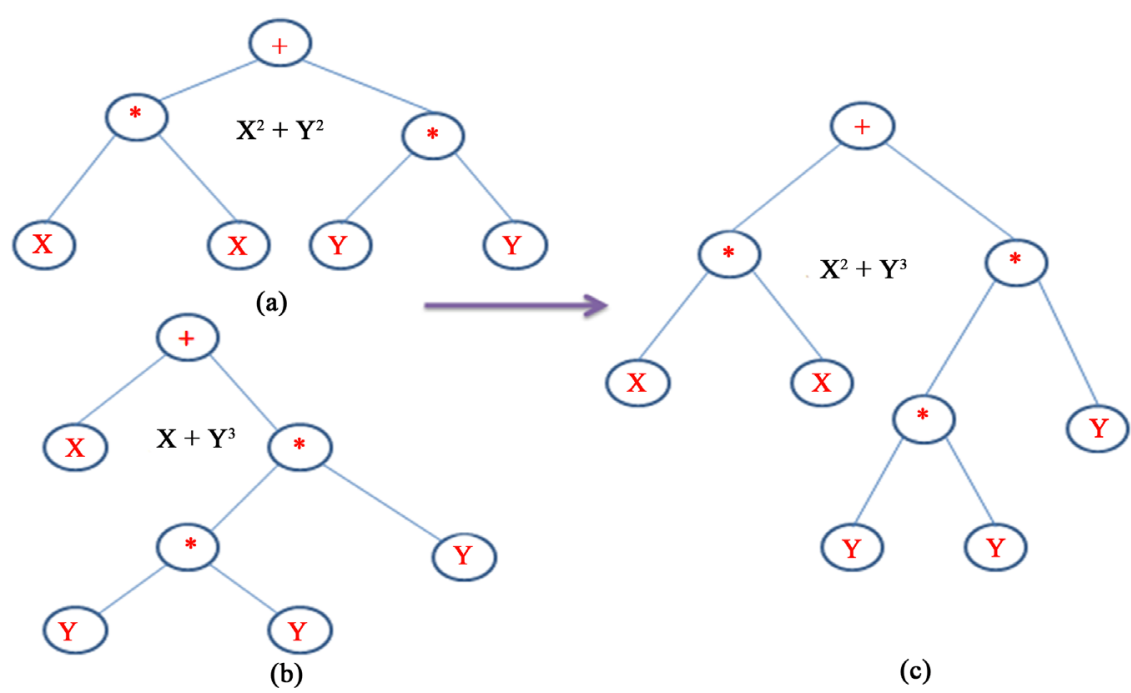

Figure 1. Using the crossover operator to create a new S-expression.

\section{GP Implementation}

The GP uses the same fundamental flow as the traditional genetic algorithm. The population of potential solutions is initialized randomly and then their fitness computed (through a simulation of executed instructions with the stack). Selection of members that can propagate into the next generation can then occur through fitness proportionate selection. With this method, the higher fit the individual, the higher the probability that they will be selected for recombination in the next generation. Evolutionary algorithms borrow concepts from Darwinian natural selection as a means to evolve solutions to problems, choosing from more fit individuals to propagate to future generations [1]-[7].

The chromosome, or program to be evolved, is made up of genes, or individual instructions. The chromosome can also be of different lengths, assigned at creation, and then inherited during the evolution.

All methods of evolutionary computation (and then GP) work as follows: create a population of individuals, evaluate their fitness, generate a new population by applying genetic operators (Cross-over, mutation and reproduction), and repeat this process a number of times as shown in Figure 2.

\section{Results and Discussion}

We have performed the GP modeling of the inclusive reaction,

$$
\begin{gathered}
\mathrm{p}^{-}+\mathrm{Ar}, \mathrm{Xe}, \mathrm{pb} \rightarrow \pi^{ \pm}+\mathrm{X} \\
\mathrm{p}+\mathrm{pb} \rightarrow \text { charged particles }
\end{gathered}
$$

using the experimental data [29]-[32] at 100 and $200 \mathrm{GeV} / \mathrm{c}$ and have done so many runs to select the best runs of the GP program, the first runs are for simulating the rapidity distribution $\frac{1}{N} \frac{\mathrm{d} N}{\mathrm{~d} Y}$ of negative pions for $\mathrm{p}^{-}-\mathrm{Au}, \mathrm{Ag}, \mathrm{Mg}$ collisions at $100 \mathrm{GeV} / \mathrm{c}$. They were configured to have the lab momentum $\left(P_{\mathrm{Lab}}\right)$, mass number (A) and the number of particles per unit solid angle (Y) as inputs and the output is the corresponding rapidity distribution $\left(\frac{1}{N} \frac{\mathrm{d} N}{\mathrm{~d} Y}\right)$ of negative pions at the given momentum as shown in Figure 3.

The second ones are for simulating the rapidity distribution $\left(\frac{1}{N} \frac{\mathrm{d} N}{\mathrm{~d} Y}\right)$ of positive pions for $\mathrm{p}^{-}$-Au, $\mathrm{Ag}, \mathrm{Mg}$ collisions at $100 \mathrm{GeV} / \mathrm{c}$ as an output and the inputs are the same as in Figure 3.

The third ones are for simulating the rapidity distribution $\left(\frac{1}{N} \frac{\mathrm{d} N}{\mathrm{~d} Y}\right)$ of positive pions for $\mathrm{p}^{-}-\mathrm{Ar}, \mathrm{p}^{-}-\mathrm{Xe}$ 


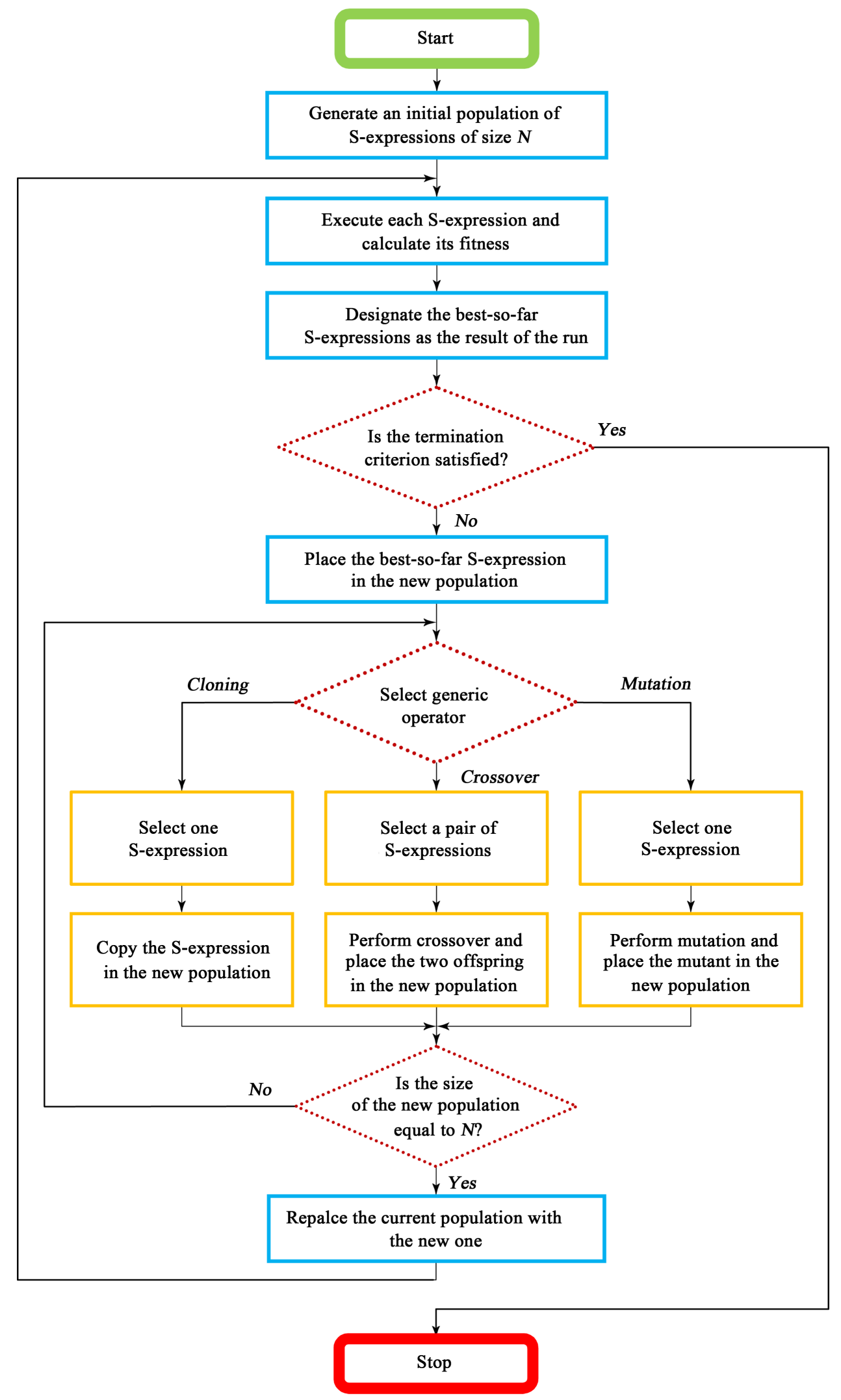

Figure 2. Flowchart for GP. 


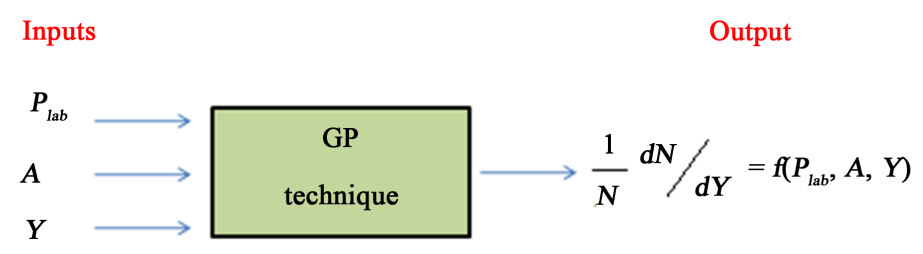

Figure 3. A block diagram of the GP technique.

collisions at $200 \mathrm{GeV} / \mathrm{c}$ as an output and the inputs are the same as in Figure 3.

The fourth ones are for simulating the rapidity distribution $\left(\frac{1}{N} \frac{\mathrm{d} N}{\mathrm{~d} Y}\right)$ of negative pions for $\mathrm{p}^{-}-\mathrm{Ar}, \mathrm{p}^{-}-\mathrm{Xe}$ collisions at $200 \mathrm{GeV} / \mathrm{c}$ as an output and the inputs are the same as in Figure 3.

The fifth ones are for simulating the rapidity distribution $\left(\frac{1}{N} \frac{\mathrm{d} N}{\mathrm{~d} Y}\right)$ of charged pions for $\mathrm{p}^{-}-\mathrm{Ar}, \mathrm{p}^{-}-\mathrm{Xe}$ collisions at $200 \mathrm{GeV} / \mathrm{c}$ as an output and the inputs are the same as in Figure 3.

The last ones are for simulating the rapidity distribution $\left(\frac{1}{N} \frac{\mathrm{d} N}{\mathrm{~d} Y}\right)$ of charged particles for $\mathrm{p}$-pb collisions at $5.02 \mathrm{TeV}$ as an output and the inputs are the same as in Figure 3.

According to all the runs, we have obtained the corresponding tree and their equivalent discovered functions Equations (2)-(7) generated for the rapidity distribution $\left(\frac{1}{N} \frac{\mathrm{d} N}{\mathrm{~d} Y}\right)$ of total charged, positive and negative pions for $\mathrm{p}^{-}$-Ar collisions at $100,200 \mathrm{GeV} / \mathrm{c}$ and $5.02 \mathrm{TeV}$.

The output, the rapidity distribution $\left(\frac{1}{N} \frac{\mathrm{d} N}{\mathrm{~d} Y}\right)$, as a function of the inputs $\left(P_{L a b}, A, Y\right)$ is given as follows:

For negative pions at $100 \mathrm{GeV} / \mathrm{c}$,

$$
\frac{1}{N} \frac{\mathrm{d} N}{\mathrm{~d} Y}=\left[\sin ^{2}(F)-\sin \left(\mathrm{e}^{E}\right)\right]
$$

For positive pions at $100 \mathrm{GeV} / \mathrm{c}$,

$$
\frac{1}{N} \frac{\mathrm{d} N}{\mathrm{~d} Y}=\mathrm{e}^{\left[0.8613 \sin \left(X_{1}\right) / R\right]+E}
$$

For positive pions at $200 \mathrm{GeV} / \mathrm{c}$,

$$
\frac{1}{N} \frac{\mathrm{d} N}{\mathrm{~d} Y}=\sin (H+C)+\mathrm{e}^{J}
$$

For negative pions at $200 \mathrm{GeV} / \mathrm{c}$,

$$
\frac{1}{N} \frac{\mathrm{d} N}{\mathrm{~d} Y}=\sin \left\{X_{1}\left[\sin \left(\cos ^{2}(10)\right)\right]\right\}+J
$$

For charged pions at $200 \mathrm{GeV} / \mathrm{c}$,

$$
\frac{1}{N} \frac{\mathrm{d} N}{\mathrm{~d} Y}=\sin ^{4}\left(0.62932 X_{1}\right)+b
$$

For charged particles at $5.02 \mathrm{TeV}$,

$$
\frac{1}{N} \frac{\mathrm{d} N}{\mathrm{~d} Y}=E+U
$$

For more details about $F, E, H$, $U$, etc., see Appendices 1-6.

The comparison between the pions rapidity distribution $\left(\frac{1}{N} \frac{\mathrm{d} N}{\mathrm{~d} Y}\right)$ computed by employing our discovered functions Equations (2)-(7) and the corresponding experimental data [29]-[31] are represented in Figure 4 for negative pions for $\mathrm{p}^{-}-\mathrm{Au}, \mathrm{Ag}, \mathrm{Mg}$ collisions at $100 \mathrm{GeV} / \mathrm{c}$, Figure 5 for positive pions for $\mathrm{p}^{-}-\mathrm{Au}, \mathrm{Ag}, \mathrm{Mg}$ 
collisions at $100 \mathrm{GeV} / \mathrm{c}$, Figure 6 for positive pions for $\mathrm{p}^{-}-\mathrm{Ar}$ (the GP model cannot describe the data when the axial value is near 0 because the noisy behavior of data around the axial), $\mathrm{p}^{-}-\mathrm{Xe}$ collisions at $200 \mathrm{GeV} / \mathrm{c}$. Figure 7 for negative pions for $\mathrm{p}^{-}-\mathrm{Ar}, \mathrm{p}^{-}$-Xe collisions at $200 \mathrm{GeV} / \mathrm{c}$, Figure 8 for charged pions for $\mathrm{p}^{-}-\mathrm{Ar}, \mathrm{p}^{-}$-Xe collisions at $200 \mathrm{GeV} / \mathrm{c}$, Figure 9 for charged particles for $\mathrm{p}-\mathrm{pb}$ collisions at $5.02 \mathrm{TeV}$.

In order to generate the GP model we have implemented the GP steps (fitness evaluation, reproduction, crossover and mutation) that were mentioned in Section 3. Our six discovered functions are generated using the obtained control GP parameters, which are shown in Table 1.
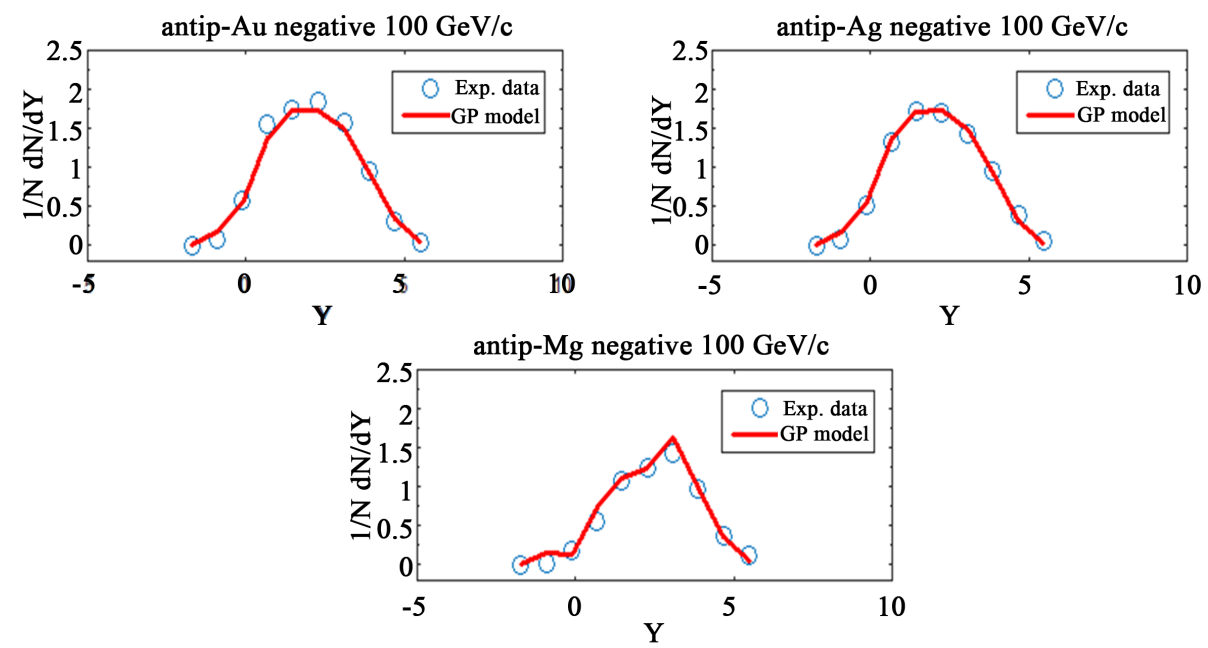

Figure 4. The discovered rapidity distribution of negative pions $\left(\frac{1}{N} \frac{\mathrm{d} N}{\mathrm{~d} Y}\right)$ for antip-Au, antip-Ag, antip-Mg interaction at $100 \mathrm{GeV} / \mathrm{c}$ ( (-) GP model, (o) experimental data.
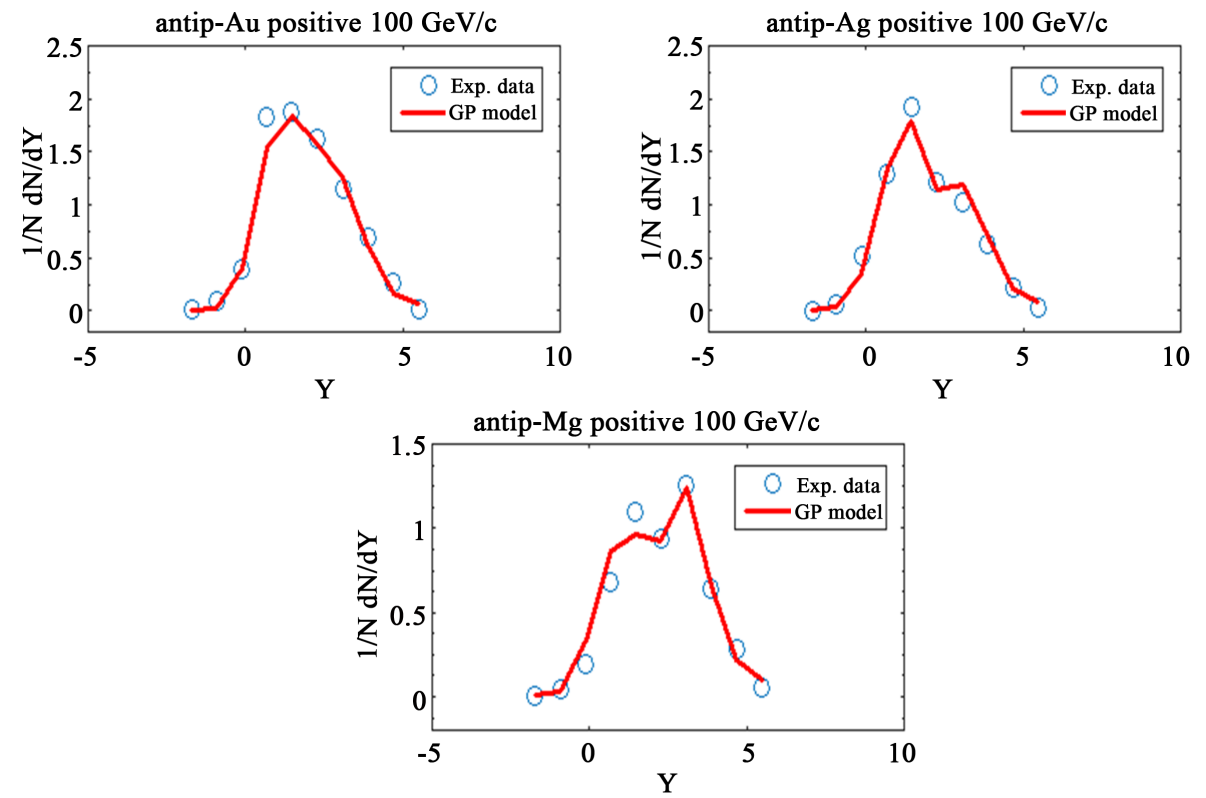

Figure 5. The obtained rapidity distribution of positive pions $\left(\frac{1}{N} \frac{\mathrm{d} N}{\mathrm{~d} Y}\right)$ for antip-Au, antip-Ag, antip-Mg interaction at $100 \mathrm{GeV} / \mathrm{c}$ : (-) GP model, (o) experimental data. 

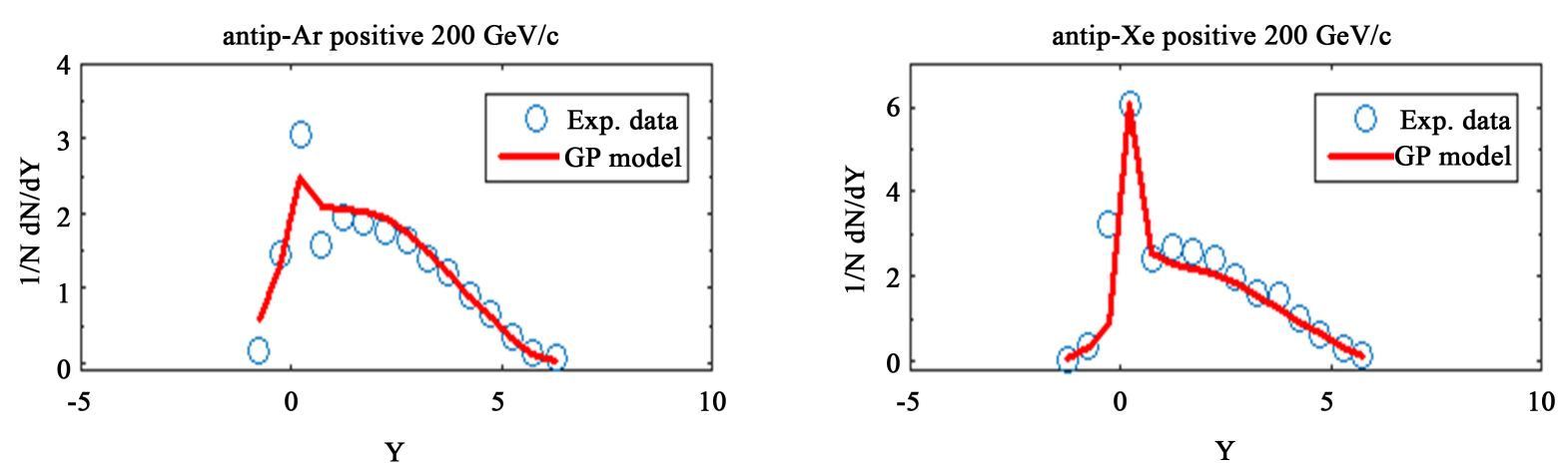

Figure 6. The discovered rapidity distribution of negative pions $\left(\frac{1}{N} \frac{\mathrm{d} N}{\mathrm{~d} Y}\right)$ for antip-Au, antip-Ag, antip-Mg interaction at $100 \mathrm{GeV} / \mathrm{c}$ : (-) GP model, (o) experimental data.
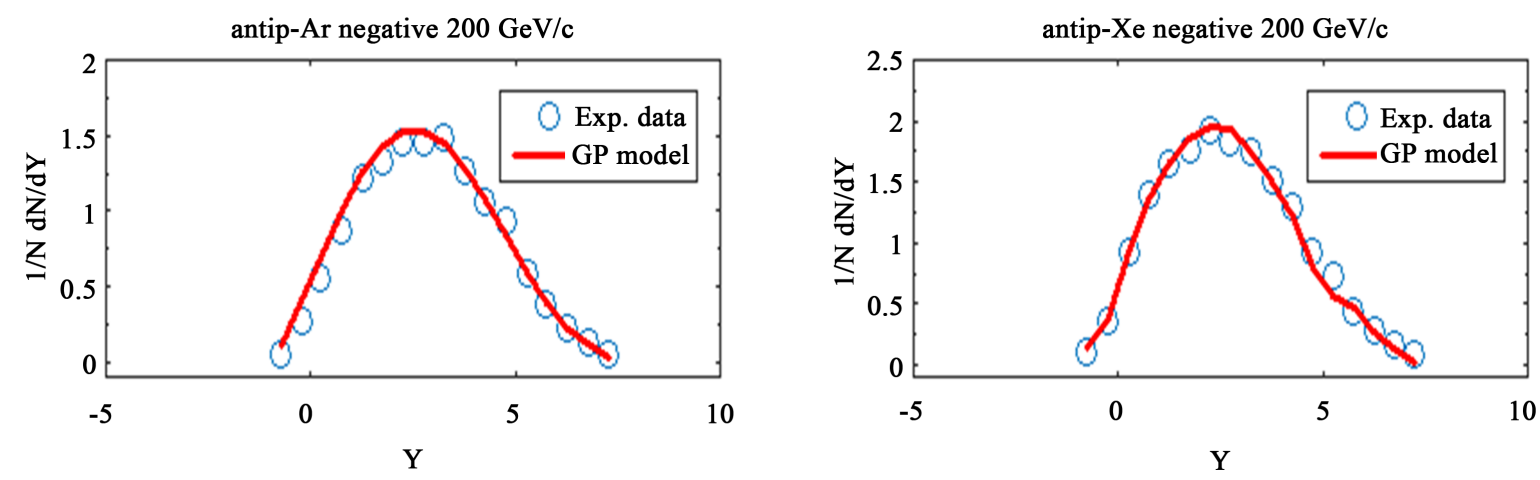

Figure 7. GP-simulated for rapidity distribution of negative pions $\left(\frac{1}{N} \frac{\mathrm{d} N}{\mathrm{~d} Y}\right)$ for antip-Ar, antip-Xe at $200 \mathrm{GeV} / \mathrm{c}$ : ( -) GP model, (o) experimental data.

Table 1. Optimal parameters controlling GP program.

\begin{tabular}{|c|c|c|c|}
\hline GP parameters & Total charged pions/particles & Positive pions & Negative pions \\
\hline Individuals & 1000 & 1000 & 1000 \\
\hline Generations & 50 & 50 & 50 \\
\hline Function set & $\begin{array}{l}+,-, *, /, \ln , \text { log, sin, } \\
\text { cos, tan, sqrt, exp, power }\end{array}$ & $\begin{array}{l}+,-,, *, /, \ln , \log , \text { sin, } \\
\text { cos, tan, sqrt, exp, power }\end{array}$ & $\begin{array}{c}+,-, *, /, \ln , \log , \text { sin, } \\
\text { cos, tan, sqrt, exp, power }\end{array}$ \\
\hline Terminal set & $P_{L a b}, A$ and $Y$ & $P_{L a b}, A$ and $Y$ & $P_{L a b}, A$ and $Y$ \\
\hline Fitness function & MSE & MSE & MSE \\
\hline Selection & Tournament & Tournament & Tournament \\
\hline Fitness at $5.02 \mathrm{TeV}$ & 0.9673 & - & - \\
\hline Mutation rate & 0.01 & - & - \\
\hline Crossover rate & 0.9 & - & - \\
\hline Fitness at $200 \mathrm{GeV} / \mathrm{c}$ & 4.9503 & 6.8111 & 1.8313 \\
\hline Mutation rate & 0.9 & 0.01 & 0.01 \\
\hline Crossover rate & 0.01 & 0.9 & 0.9 \\
\hline Fitness at $100 \mathrm{GeV} / \mathrm{c}$ & - & 2.178635 & 1.5753 \\
\hline Mutation rate & - & 0.01 & 0.9 \\
\hline Crossover rate & - & 0.9 & 0.01 \\
\hline
\end{tabular}



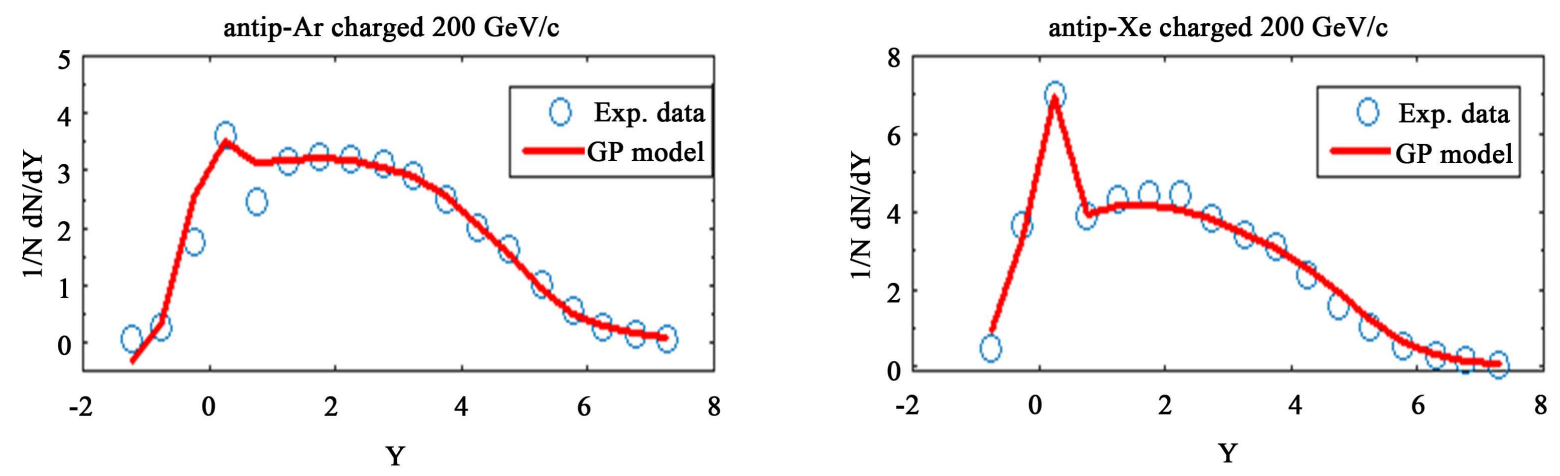

Figure 8. Simulated results for rapidity distribution of chared pions $\left(\frac{1}{N} \frac{\mathrm{d} N}{\mathrm{~d} Y}\right)$ for antip-Ar, antip-Xe at $200 \mathrm{GeV} / \mathrm{c}$ : (一) GP model, (o) experimental data.

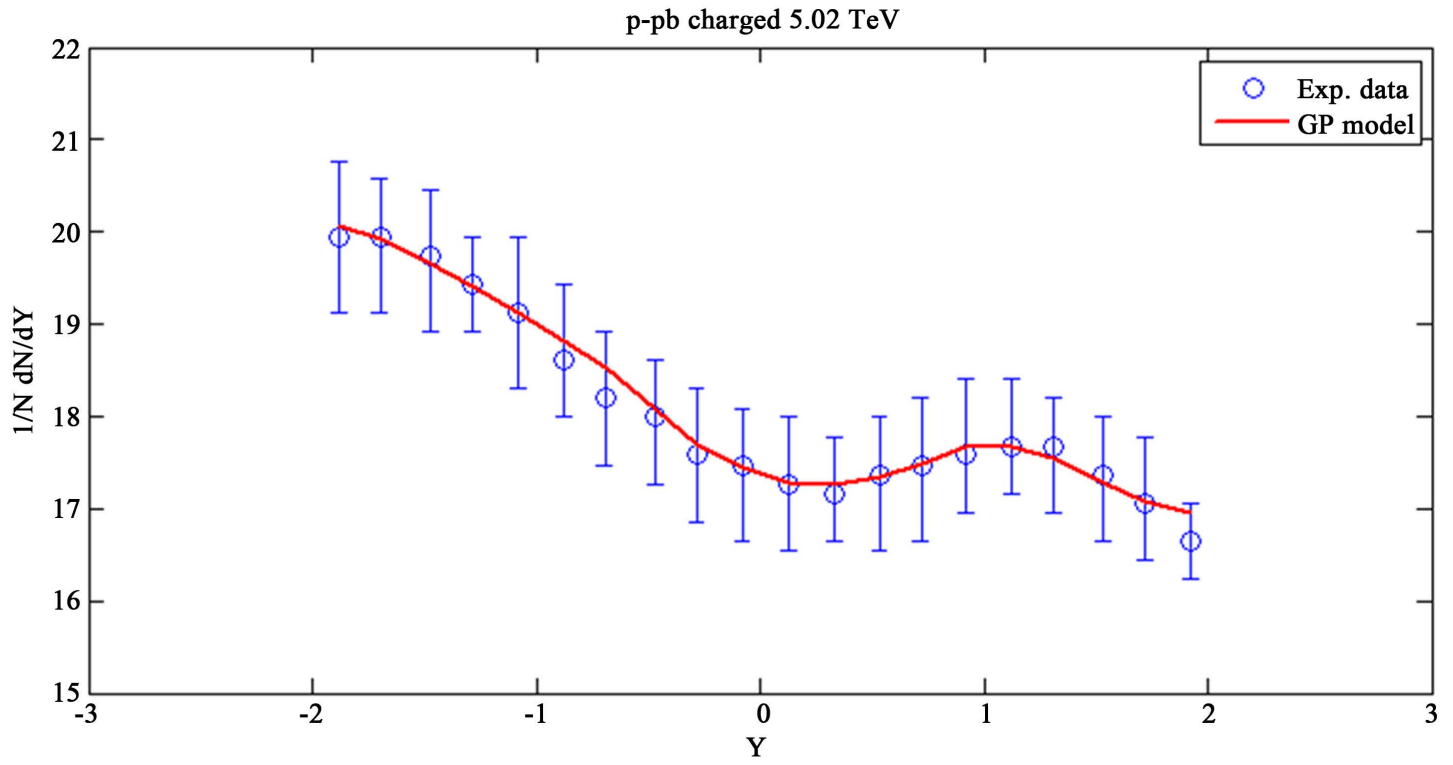

Figure 9. The discovered rapidity distribution of charged particles for p-Pb interaction at $5.02 \mathrm{TeV}$ : (一) GP model, (o) experimental data.

The statistical error criterion of mean square error (MSE) was used to measure the deviation between the experimental (actual) and simulated values. The statistical parameter MSE has been used in this work as a performance metric [33] to compare the GP simulation with the actual observations (experimental data) and these were evaluated by using Matlab program. The smaller the values of MSE the closer the simulated values to the experimental ones. Our obtained MSE values for the seven discovered functions are given in Table 1, which show also that the performance of the GP model is clearly suitable.

\section{Conclusions}

GP model has been shown to be a vital method for modeling the h-A interactions. The current article presents an efficient approach for computing the rapidity distribution $\left(\frac{1}{N} \frac{\mathrm{d} N}{\mathrm{~d} Y}\right)$ of charged, positive and negative pions for $\mathrm{p}^{-}-\mathrm{Ar}$ and $\mathrm{p}^{-}-\mathrm{Xe}$ collisions at $200 \mathrm{GeV} / \mathrm{c}$ and charged particles for $\mathrm{p}-\mathrm{pb}$ collisions at $5.02 \mathrm{TeV}$ through the obtained discovered functions. All the discovered functions show a clear and excellent match to the experimental data. 
The interaction of hadrons with atomic nuclei at high and ultrahigh energies is an issue of great importance since in-depth studies about it provide the necessary information on properties which cannot be examined by analyzing only hadron-hadron interactions.

Finally, the present work has proved that the GP approach can be employed effectively to model the h-A interactions at the given energy.

\section{References}

[1] Jones, M.T. (2008) Artificial Intelligence: A Systems Approach Infinity. Science Press LLC, Hingham.

[2] Banzhaf, W., et al. (1998) Genetic Programming: An Introduction: On the Automatic Evolution of Computer Programs and Its Applications. Morgan Kaufmann, Burlington.

[3] Higuchi, T., Liu, Y. and Yao, X. (2006) Evolvable Hardware. Genetic and Evolutionary Computation. http://dx.doi.org/10.1007/0-387-31238-2

[4] EvoNews Professor Hans-Paul Schwefel Talks to EvoNews (1999) Available Online. http://evonet.lri.fr/evoweb/news_events/news_features/

[5] Fogel, L.J., Owens, A.J. and Walsh, M.J. (1966) Artificial Intelligence through Simulated Evolution. Wiley, New York.

[6] Levenick, J.R (1991) Inserting Introns Improves Genetic Algorithm Success Rate: Taking a Cue from Biology. Proceedings on the 4th International Conference on Genetic Algorithms.

[7] Rechenberg I (1965) Cybernetic Solution Path of an Experimental Problem Technical Report Library Translation No. 1122. Royal Aircraft Establishment, Farnborough.

[8] Zheng, S.J., Zhang, N., Xia, Y.J. and Wang, H.T. (2014) Research on Non-Uniform Strain Profile Reconstruction along Fiber Bragg Grating via Genetic Programming Algorithm and Interrelated Experimental Verification. Optics Communications, 315, 338-346.

[9] Koza, J.R. (1992) Genetic Programming: On the Programming of Computers by Means of Natural Selection. The MIT Press, Cambridge.

[10] Koza, J.R. (1990) Genetic Programming: A Paradigm for Genetically Breeding Populations of Computer Programs to Solve Problems. Stanford University Computer Science Department Technical Report STAN-CS-90-1314.

[11] Holland, J.H. (1975) Adaptation in Natural and Artificial Systems. University of Michigan Press, Ann Arbor.

[12] Tantawy, M., El-Mashad, M. and El-Bakry, M.Y. (1998) Multiparticle Production Process in High Energy NucleusNucleus Collisions. Indian Journal of Physics, 72A, 73-82.

[13] Moussa, M.A., El-Bakry, M.Y., Radi, A., El-Dahshan, E.-S.A., Habashy, D.M. and Abbas, E.G. (2012) Topological Cross Sections and Multiplicity Distributions for and Interactions at High Energies. International Journal of Scientific and Engineering Research, 3.

[14] Fermi, E. (1950) High Energy Nuclear Events. Progress of Theoretical Physics, 5, 570-583. http://dx.doi.org/10.1143/ptp/5.4.570

[15] Fermi, E. (1951) Angular Distribution of the Pions Produced in High Energy Nuclear Collisions. Physical Review, 81, 683-687. http://dx.doi.org/10.1103/PhysRev.81.683

[16] Ranft, J. (1970) Secondary Particle Production According to the Thermodynamical Model and New Experimental Data. Physics Letters B, 31, 529-532. http://dx.doi.org/10.1016/0370-2693(70)90082-1

[17] Xu, C., Chao, W.-Q., Meng, T.-C. and Huang, C.-S. (1986) Statistical Approach to Nondiffractive Hadron-Hadron Collisions: Multiplicity Distributions and Correlations in Different Rapidity Intervals. Physical Review D, 33, 12871299. http://dx.doi.org/10.1103/PhysRevD.33.1287

[18] Nambu, Y. (1976) The Confinement of Quarks. Scientific American, 235, 48-61. http://dx.doi.org/10.1038/scientificamerican1176-48

[19] Gyulassy, M. (1985) Introduction to QCD Thermodynamics and the Quark-Gluon Plasma. Progress in Particle and Nuclear Physics, 15, 403-442. http://dx.doi.org/10.1016/0146-6410(85)90076-6

[20] Kisslinger, L.S. (1985) Nuclear Physics and Quark/Gluon QCD. Nuclear Physics A, 446, 479-488. http://dx.doi.org/10.1016/0375-9474(85)90624-4

[21] Jacob, M. and Slansky, R. (1972) Nova Model of Inclusive Reactions. Physical Review D, 5, 1847-1870. http://dx.doi.org/10.1103/PhysRevD.5.1847

[22] Hwa, R.C. (1970) Bootstrap Model for Diffractive Processes: Complementarity of the Yang and Regge Models. Physical Review D, 1, 1790-1809. http://dx.doi.org/10.1103/PhysRevD.1.1790 
[23] Hwa, R.C. (1971) Multiplicity Distribution and Single-Particle Spectrum in the Diffractive Model. Physical Review Letters, 26, 1143-1147. http://dx.doi.org/10.1103/PhysRevLett.26.1143

[24] EL-Bakry, S.Y., El-Dahshan, E.-S. and EL-Bakry, M.Y. (2011) Total Cross Section Prediction of the Collisions of Positrons and Electrons with Alkali Atoms Using Gradient Tree Boosting. Indian Journal of Physics, 85, 1405-1415.

[25] El-Bakry, M.Y. (2003) Feed Forward Neural Networks Modeling for K-P Interactions. Chaos, Solitons and Fractals, 18, 995-1000. http://dx.doi.org/10.1016/S0960-0779(03)00068-7

[26] El-Bakry, M.Y. (2004) A Study of K-P Interaction at High Energy Using Adaptive Fuzzy Inference System Interactions. International Journal of Modern Physics C, 15, 1013-1020. http://dx.doi.org/10.1142/S0129183104006467

[27] El-Bakry, M.Y., El-Dahshan, E., Radi, A., Tantawy, M. and Moussa, M.A. (2013) A Genetic Programming for Modeling Hadron-Nucleus Interactions at $200 \mathrm{GeV} / \mathrm{c}$. International Journal of Scientific and Engineering Research, 4, 7.

[28] Ghosh, D. (1983) International Conference on Cosmic Ray 08.

[29] De Marzo, C., De Palma, M., Distante, A., et al. (1982) Multiparticle Production on Hydrogen, Argon, and Xenon Targets in a Streamer Chamber by 200-GeV/c Proton and Antiproton Beams. Physical Review D, 26, 1019-1035. http://dx.doi.org/10.1103/PhysRevD.26.1019

[30] Arneodo, M., Arvidson, A., Aubert, J.J., et al. (1987) Comparison of Multiplicity Distributions to the Negative Binomial Distribution in Muon-Proton Scattering. Zeitschrift für Physik C Particles and Fields, 35, 335-345. http://dx.doi.org/10.1007/BF01570769

[31] Kittle, W. (1973) Combining Inclusive and Exclusive Data Analyses-What Have We Learned So Far? Journal of Physics A: Mathematical, Nuclear and General, 6, 733.

[32] Abelev, B., et al., ALICE Collaboration (2013) Pseudorapidity Density of Charged Particles in p + Pb Collisions at s N N=5.02 TeV. Physical Review Letters, 110, Article ID: 032301. http://dx.doi.org/10.1103/PhysRevLett.110.032301

[33] Hong, W.-C. (2008) Rainfall Forecasting by Technological Machine Learning Models. Applied Mathematics and Computation, 200, 41-57. http://dx.doi.org/10.1016/j.amc.2007.10.046 


\section{Appendices}

1. Rapidity Distribution of Negative Pions for $\mathbf{p}^{-}-\mathrm{Au}, \mathrm{p}^{-}-\mathrm{Ag}$ and $\mathrm{p}^{-}-\mathrm{Mg}$ Interaction at $100 \mathrm{GeV} / \mathrm{c}$

$$
\begin{gathered}
A=\sin ^{2}\left(10-\frac{X_{1}+X_{3}}{1.2414}\right), \quad B=A\left\{\log _{10}\left[\cos ^{2}\left(X_{1}+\mathrm{e}^{X_{3}}\right)\right]\right\}, \quad C=\sin ^{2}\left(10-\frac{X_{1}+0.60169}{1.2414}\right), \\
D=\log _{10}\left[\cos \left(C+\sin \left(e^{B}\right)\right)\right], \quad E=\frac{0.17 D}{X_{1} \mathrm{e}^{\sin \left(X_{3}\right)}}, \quad F=10-\left[\frac{X_{1}+0.60169}{1.2414}\right]
\end{gathered}
$$

2. Rapidity Distribution of Positive Pions for $\mathrm{p}^{-}-\mathrm{Au}, \mathrm{p}^{-}-\mathrm{Ag}$ and $\mathrm{p}^{-}-\mathrm{Mg}$ Interaction at $100 \mathrm{GeV} / \mathrm{c}$

$$
\begin{gathered}
A=X_{2}-X_{1}-\mathrm{e}^{X_{1}}+X_{3}-970.8738, \quad B=X_{2}-0.12013-\tan (A), \quad C=(10 / B)^{\sin \left(X_{3}\right)}-\cos \left(X_{3}\right), \\
D=\cos ^{2}(0.187454+C), \quad E=\sin ^{2}\left(0.550985 X_{1}\right)-D, \\
F=\cos \left(\log _{10}\left(\tan \left(X_{3}\right)\right)+\left(\tan \left(\mathrm{e}^{X_{3}}\right)-\cos \left(X_{3}\right)\right)\right), \\
G=\tan \left(X_{3}\right)+X_{3} X_{2}-\left(X_{1}+10 / X_{2}\right), \quad H=\sin \left[(G)^{10} / F\right], \quad I=\frac{-2.91765}{10 / X_{3}}, \\
J=\frac{\left(10 / X_{3}\right)^{I}-0.82908+H}{X_{3}}, K=\log _{10}\left(\tan \left(X_{3}\right)\right)+\cos \left(X_{3}\right)-\tan \left(\mathrm{e}^{X_{3}}\right), \\
L=X_{2}^{X_{3}}+X_{3} X_{2}-10, \quad M=\left[\frac{X_{1}+10}{X_{2}}\right]^{\sin \left(X_{3}\right)}-0.82908, \quad N=\sin \left[\frac{-3.64929}{10 / X_{3}}\right], \\
O=\cos \left[\sin \left(10 / X_{3}\right)^{N}+m\right], \quad P=\sin \left(\frac{\cos (K)}{L^{O}}\right)+J^{I}-0.8298, Q=\sin \left(0.176 X_{1}\right)-\cos ^{3}(P), \quad R=\tan \left(\mathrm{e}^{Q}\right)
\end{gathered}
$$

3. Rapidity Distribution of Positive Pions for $\mathrm{p}^{-}-\mathrm{Xe}$ and $\mathrm{p}^{-}$-Ar Interaction at $200 \mathrm{GeV} / \mathrm{c}$

$$
\begin{gathered}
A=\frac{1.228815}{\left[X_{1} / 0.831998\right]}, \quad B=\frac{\mathrm{e}^{\sin (A)}}{X_{1} / 0.724939}, \quad C=X_{1} \cos \left[0.984928 \sin \left(\mathrm{e}^{\sin (B)}\right)\right], \\
D=\frac{\left[10 / \cos \left(\sin \left(X_{1}\right)\right)\right]}{\left[56.2934-\log _{10}\left(X_{1}\right)\right]}, \quad E=\sin \left(X_{1}\right)-\cos ^{2}\left[(D-10) \log _{10}\left(X_{1}\right)\right], \\
G=0.984928 \sin \left(\mathrm{e}^{\sin (A)}\right), \quad H=0.984928 \sin ^{2}(G+E), \quad I=0.942092 \cos \left(X_{2}\right), \quad J=\frac{\mathrm{e}^{I}}{\left(X_{1} / 0.20605\right)}
\end{gathered}
$$

4. Rapidity Distribution of Negative Pions for $\mathrm{p}^{-}$-Ar and $\mathrm{p}^{-}$-Xe Interaction at $200 \mathrm{GeV} / \mathrm{c}$

$$
\begin{gathered}
A=X_{3} /\left[10 X_{1} / 10 \mathrm{e}^{X_{1}+\cos \left(X_{2}\right)}\right], \quad B=\sin \left[X_{1}\left(0.995888+\frac{10 X_{1}}{0.39473}\right)\right] \\
C=\mathrm{e}\left(\mathrm{e}^{X_{1}+0.66922}\right)-\cos ^{2}\left(X_{3}\right), \quad D=10\left[\frac{X_{1}}{\mathrm{e}^{X_{2}}}\right] / 0.39473, \quad E=\left(C-\frac{X_{3}}{D}\right) / B, \\
G=\cos ^{2}[\sin (F)]-X_{3} /\left(\frac{10 X_{1}}{0.39473}\right), \quad H=\log _{10}(G)-\left(X_{3} / \frac{10 X_{1}}{0.39473}\right), \\
I=\sin \left[\mathrm{e}^{\sin \left(X_{2}\right)}\right] / X_{1}, \quad J=\cos \left[\sin \left(\frac{\mathrm{e}^{\sin \left(X_{3}\right)} X_{1}}{I}\right)\right]
\end{gathered}
$$


5. Rapidity Distribution of Charged Pions for $\mathrm{p}^{-}$-Xe and $\mathrm{p}^{-}$-Ar Interaction at $200 \mathrm{GeV} / \mathrm{c}$

$$
\begin{aligned}
& A=\left\{\left[\sin \left(X_{1} X_{2}\right)\right]^{X_{3}}-\frac{X_{1}}{0.9331}\right\}^{0.13958}, B=\log _{10}\left[\frac{A^{7.6003}-\left(\frac{X_{2}}{X_{1}}\right)}{0.533419}\right], C=\sin ^{2}\left(0.62932 X_{1}\right)+B, \\
& D=\sin \left[\sin ^{2}\left(0.62932 X_{1}\right)-0.37885\right], E=X_{2}^{0.13958}-\left[\frac{\frac{X_{2}}{X_{1}}+X_{2}}{X_{1}}\right], \quad F=\sin ^{2}\left(0.62932 X_{1}\right)+\log _{10}\left(\frac{E}{D}\right), \\
& G=\left\{[\sin (F)]^{C}-1\right\}^{0.13958}, H=\left(X_{2}^{0.88504}+X_{2}\right)^{0.76003}, I=\left[\frac{G^{H}-\left(\frac{X_{2}}{X_{1}}\right)}{0.533419}\right], \\
& J=\sin \left[\sin ^{2}\left(0.62932 X_{1}\right)+\log _{10}(I)\right], \quad K=\sin \left(0.62932 X_{1}\right)-\tan \left[\sin \left(X_{2}\right)\right], \\
& L=0.841471 \cos \left(X_{1}\right)^{K}, M=\left\{L-\sin \left[\cos \left(\frac{X_{3}}{0.88504 X_{2}}\right)+\cos \left(X_{3}^{2}\right)\right]\right\}^{0.13958}, \\
& N=M-\frac{\frac{X_{2}}{X_{1}}+X_{2}}{X_{1}}, \quad O=\frac{N}{\sin \left[\sin ^{2}\left(0.62932 X_{1}\right)+\log _{10}(0.41797)\right]}, \\
& P=\sin \left[\sin ^{2}\left(0.62932 X_{1}\right)+\log _{10}(O)\right], Q=\left\{\left[\sin \left(X_{2} X_{1}\right)\right]^{X_{3}}-\frac{X_{1}}{0.9331}\right\}^{0.13958}, \\
& R=\left\{\left[\sin \left(X_{3}\right)\right]+X_{2}^{0.88504}\right\}^{0.76003}, S=\log _{10}\left\{Q^{R}-\frac{X_{2} / X_{1}}{0.533419}\right\}^{0.13958}, \\
& T=\sin ^{2}\left(0.62932 X_{1}\right)+S, \quad U=\mathrm{e}^{\frac{\log _{10}\left(X_{3}\right)+0.010309}{X_{3}+X_{2} X_{3}}}, V=\left[\cos \left(P^{T}\right)\right]^{U}, \\
& W=\sin \left\{10\left[\frac{X_{3}}{\frac{\mathrm{e}^{X_{1}}}{-0.04686}+\cos \left(X_{3}^{2}\right)}\right]\right\}, \quad X=\left\{\left[\sin \left(X_{1} X_{3}\right)\right]^{X_{3}}-1\right\}^{0.13958}, \\
& Y=\left\{\left[X_{1}-\sin \left(X_{2}\right)\right]^{X_{3}}+X_{2}^{0.88504}\right\}^{0.76003}, Z=\left(V W-X_{1}\right)^{\sin \left(X^{Y}\right)}, \\
& a=\left[Z-\frac{\left(X_{2} / X_{1}\right)+X_{2}}{X_{1}}\right] / J, \quad b=\sin ^{2}\left(0.62932 X_{1}\right)+\log _{10}(a)
\end{aligned}
$$

\section{Rapidity Distribution of Charged Particles for p-pb Interaction at $5.02 \mathrm{TeV}$}

$$
\begin{gathered}
A=(-0.05142) \frac{\left(10-x_{1}\right)}{0.949882}, B=-9.64173 \cos \left(X_{1}\right), C=(0.786251)^{B}, \quad D=C+\log _{2}\left[\sin (10)-\log _{2}(10)\right], \\
E=\frac{\log _{2}\left(\mathrm{e}^{D}\right)}{22026.47}, \quad F=\log \left(\frac{0.97842}{X_{1}} / 10\right)\left[\log _{10}\left(\mathrm{e}^{X_{1}}\right)+X_{1}\right], \quad G=1.9999, \quad H=\cos \left[\cos \left(X_{1}\right)\right]-\sqrt{G},
\end{gathered}
$$




$$
\begin{aligned}
& I=\log \left[\log _{10}(0.4439)\right]-\tan \left\{\tan \left[\log \left(X_{1}\right)-\sqrt{X_{1}}\right]\right\}, \quad J=H \tan \left(\mathrm{e}^{\log _{10}\left(\log _{10}(I)\right)}\right), \quad K=\mathrm{e}^{\log _{10}\left(X_{1}\right)-J}, \\
& L=\left(\frac{K}{0.70831}\right)^{-9.642 \cos \left(X_{1}\right)}+\log _{2}\left[\sin (10)-\log _{2}(10)\right], \quad M=\frac{\left(0.59066-\sqrt{\log \left(X_{1}\right)+10}\right)}{L}+\log \left(X_{1}\right), \\
& N=\cos (10)-\sqrt{\frac{0.259004}{X_{1}}+\log \left(X_{1}\right)}, \quad O=1.557408 N, \quad P=1.557408(O-\sqrt{m}), \quad Q=\left(\mathrm{e}^{0.671654 \tan \left(X_{1}\right) X_{1}^{2}}\right), \\
& R=\left(\mathrm{e}^{\tan \left(X_{1}\right)^{X_{1}^{2} Q}}\right), \quad S=R-\frac{\cos \left(\mathrm{e}^{\left(\frac{10}{X_{1}}\right)}\right)}{1.071185}, T=1.557408\left[\cos \left(x_{1}\right)-S\right], \quad U=\frac{\mathrm{e}^{\log _{10}\left(X_{1}\right)-T}}{\log _{2}\left[\sin (10)-\log _{2}(10)\right]}, \\
& V=U^{-9.642 \cos \left(X_{1}\right)}, \quad W=\log _{2}\left\{\log _{2}\left[\log _{2}\left(\log _{10}\left(0.98407-x_{1}-2.142536\right)\right)\right]\right\}-3.321928+V, \\
& X=\left[0.80812+\frac{8.036777}{x}\right], \quad Y=1.557408\left[\cos \left(x_{1}\right)-\sqrt{\log \left(x_{1}\right)+1}\right], \quad Z=\left[\cos \left(x_{1}\right)-\frac{\mathrm{e}^{\log _{10}\left(X_{1}\right)-Y}}{0.70831}\right] / W \text {, } \\
& a=\cos \left(X_{1}\right)-\sqrt{\log \left(X_{1}\right)+\frac{0.259004}{X_{1}}}, b=a \tan \left[\mathrm{e}^{\log \left(\frac{10}{X_{1}}\right)^{0.8901}}\right], c=b-\sqrt{\log \left(X_{1}\right)+Z}, \\
& d=c \tan \left(\mathrm{e}^{\frac{\log _{10}\left(X_{1}\right)}{0.30103}}\right), \quad e=F-\left[\log \left(\mathrm{e}^{\log _{10}(c)-P}\right)\right], \quad f=\mathrm{e}^{\left[\mathrm{e} /\left(10-X_{1}\right)\right]}
\end{aligned}
$$

where, $X_{1}$ is the number of particles per unit solid angle $(Y), X_{2}$, lab momentum $\left(P_{L a b}\right), X_{3}$, mass number (A). 